\title{
Neoadjuvant chemotherapy, radical hysterectomy and chemoradiation for stage iva cervical carcinoma
}

\author{
John P. Micha ${ }^{1}$, Howard D. Epstein ${ }^{2}$, Michael C. Roossin ${ }^{3}$, Randy Bohart ${ }^{4}$, \\ Mark A. Rettenmaier ${ }^{5}$, Bram H. Goldstein ${ }^{6}$ \\ ${ }^{1}$ Gynecologic Oncology Associates Partners, 361 Hospital Road, Suite \#422, Newport Beach, CA 92663, USA \\ ${ }^{2}$ Department of Pathology, Hoag Hospital, 361 Hospital Road, Newport Beach, CA 92663, USA \\ ${ }^{3}$ Department of Radiology, Hoag Hospital, 361 Hospital Road, Newport Beach, CA 92663, USA \\ ${ }^{4}$ Oso Home Care, 17175 Gillette Ave, Irvine, CA 92614, USA \\ ${ }^{5} 351$ Hospital Road, Suite \#506, Newport Beach, CA 92663, USA \\ ${ }^{6}$ Women's Cancer Research Foundation, Newport Beach, CA 92663, USA
}

\begin{abstract}
Summary
Despite the efficacy of chemotherapy and radiotherapy in the management of locally advanced cervical carcinoma, the overall survival rates are relatively inauspicious. We recount a case involving a 56-year-old stage IVA cervical cancer patient who underwent neoadjuvant chemotherapy, a type $\mathrm{V}$ radical hysterectomy and adjuvant chemoradiation in April 2006. The patient remained in clinical remission for five years until she developed a metastatic pulmonary nodule in her right middle lobe that originated from the primary cervical cancer. Thereafter, the patient was diagnosed with a right metastatic cardiophrenic nodule that responded favorably to chemotherapy. However, in August 2014, she presented with a metastatic posterior cranial fossa tumor from which she ultimately expired; interestingly, radiologic imaging revealed no evidence of an abdominopelvic recurrence. While a radical hysterectomy is often indicated for advanced cervical cancer patients with residual disease or those who fail chemoradiation, perhaps in select cases (e.g., stage IVA disease), initial treatment with neoadjuvant chemotherapy and surgery followed by chemoradiation may improve patient survival.
\end{abstract}

Key words: Cervical cancer; Radical hysterectomy; Chemoradiation; Outcomes.

\section{Introduction}

There were 13,240 patients in the United States who were diagnosed with cervical cancer in 2018, 4,170 of whom will eventually succumb to their disease [1]. Despite the reasonably favorable cure rates for patients with earlystage disease, the prognosis for stage IVA cervical cancer is inauspicious, with reported 5-year survival rates approaching $16 \%$ [2].

Pelvic radiation therapy, single agent or combination platinum-based chemotherapy $[3,4]$ and brachytherapy have eventuated in more beneficial outcomes for locally advanced cervical cancer compared to neoadjuvant therapy and radical hysterectomy. The former regimen is considered the standard treatment for this disease [5] although surgery following neoadjuvant chemoradiation has reportedly further accorded encouraging outcomes in patients with advanced-stage cervical cancer [6-9].

Patients diagnosed with stage IVA cervical cancer have disease involving proximal organs in the pelvis (e.g., the rectum and bladder) [10]. They are characterized as inoperable and despite currently recommended treatment, the long-term clinical outcomes are discouraging [11, 12]. Alternatively, in select patients wherein the tumor is amenable to surgical resection $[13,14]$, performing an extended radi- cal hysterectomy for stage IVA disease may be a reasonable consideration. Herein, we describe the long-term history of a stage IVA cervical cancer patient who was initially managed with neoadjuvant chemotherapy, radical hysterectomy and adjuvant chemoradiation.

\section{Case Report}

A 56-year-old, nulligravid woman with an unremarkable medical history originally presented to our gynecologic oncology service with metrorrhagia and a hematocrit of $33.7 \%$ in April 2006. Following a dilatation and curettage, pathology revealed a grade 3 squamous cell carcinoma. Subsequently, a CT scan of the chest, abdomen and pelvis exhibited a locally confined, $7 \mathrm{~cm}$ cervical tumor that infiltrated the superior portion of the vagina (Figure 1). A cystoscopy revealed the presence of bladder invasion; moreover, she was also found to have moderate left hydronephrosis and hydroureter, with decreased left renal perfusion.

The patient completed 3 cycles of paclitaxel (175 $\mathrm{mg} / \mathrm{m}^{2}$ ) and carboplatin (AUC 5) in June 2006, which reduced the tumor size to $4 \mathrm{~cm}$ in diameter although there was persistent vaginal involvement. In July 2006, the patient underwent a laparotomy, type $\mathrm{V}$ radical hysterectomy [13] with upper vaginectomy, including an en bloc resec-
Eur. J. Gynaecol. Oncol. - ISSN: 0392-2936 XLI, n. 5, 2020

doi: $10.31083 /$ j.ejgo.2020.05.2086
This is an open access article under the CC BY 4.0 license (https://creativecommons.org/licenses/by/4.0/). 


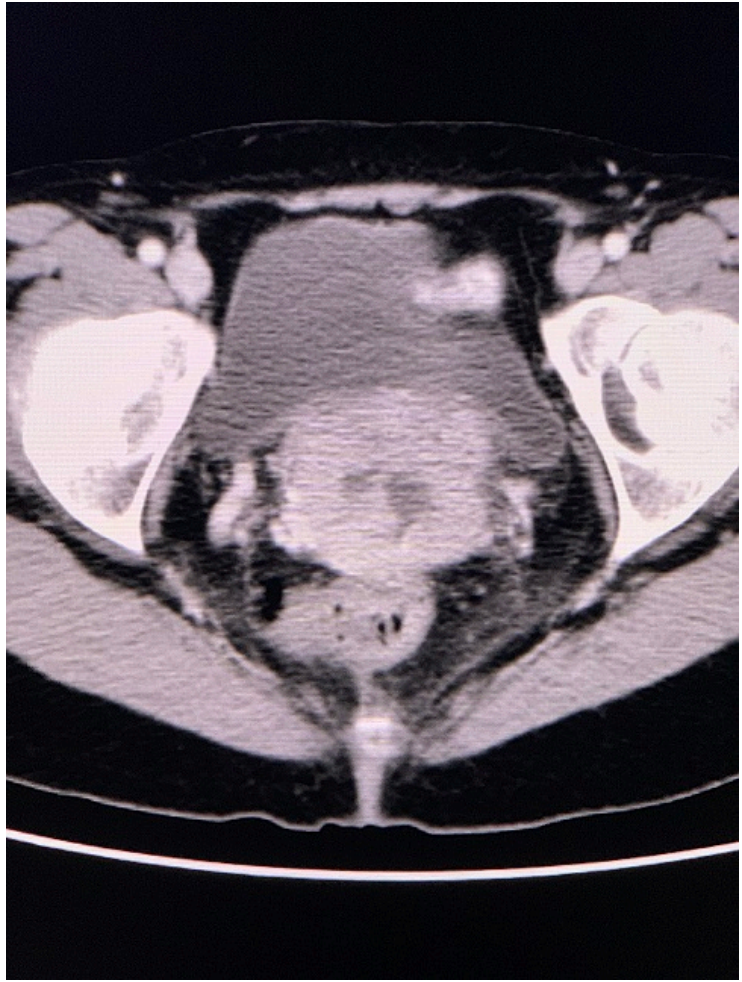

Figure 1. - April 2006 CT scan of the pelvis.

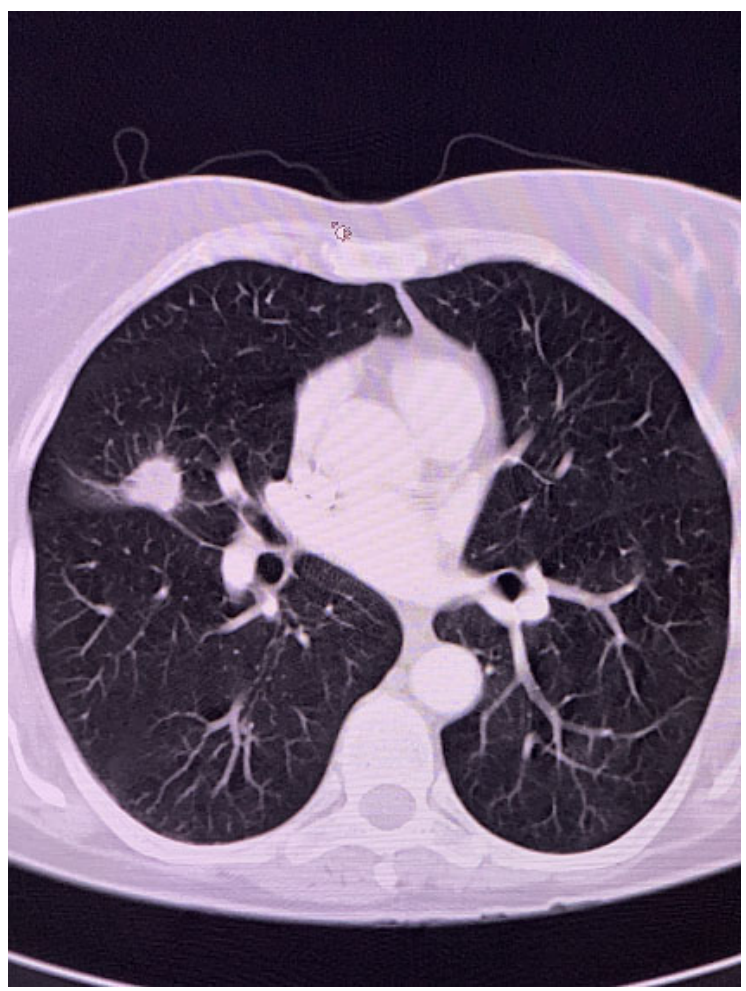

Figure 2. - October $2011 \mathrm{CT}$ scan of the chest.

tion involving the base of the bladder and distal portion of a double left ureter, double left ureteral neocystotomy with placement of two left ureteral stents, intentional ante- rior cystotomy with placement of a right ureteral stent, and bilateral pelvic and para-aortic lymphadenectomy. There were no significant intraoperative complications and estimated blood loss was $400 \mathrm{~mL}$.

There was gross tumor residuum measuring $4.5 \times 3.0 \times$ $1.0 \mathrm{~cm}$ involving the bladder mucosa; the surgical margins and resected nodes were negative. In consideration of the bladder involvement, final pathology revealed a poorly differentiated, squamous cell, stage IVA cervical carcinoma.

In September 2006, the patient commenced with 3 cycles of weekly cisplatin $\left(30 \mathrm{mg} / \mathrm{m}^{2}\right)$ chemotherapy, $50.4 \mathrm{~Gy}$ of pelvic radiotherapy and 14 Gy to the bladder, to which the disease responded favorably. In October 2009, the patient experienced chronic pain and was admitted to the Operating Room, whereupon she underwent a laparoscopic lysis of adhesions; there was neither evidence of a bowel obstruction nor tumor recurrence.

The patient remained disease free until October 2011, wherein a CT scan of the chest revealed a pulmonary nodule in her right middle lobe (Figure 2). A CT-guided biopsy of the lung lesion confirmed squamous cell carcinoma. In December 2011, the patient underwent a right middle lobe lobectomy. Pathology from the lesion corroborated invasive squamous cell carcinoma, consistent with her cervical primary. The patient subsequently underwent surveillance.

In December 2012, a right cardiophrenic mass was identified via a core biopsy, which revealed moderately differentiated squamous cell carcinoma and focal necrosis; there was no evidence of recurrent abdominal or pelvic cancer. The patient was administered 6 cycles of paclitaxel $\left(175 \mathrm{mg} / \mathrm{m}^{2}\right.$ ) and carboplatin (AUC 6) in January 2013, followed by 6 cycles of paclitaxel $\left(135 \mathrm{mg} / \mathrm{m}^{2}\right)$ maintenance chemotherapy. In December 2013, the chemotherapy was changed to bevacizumab $(10 \mathrm{mg} / \mathrm{kg})$ every 3 weeks, of which she received 6 cycles. However, in August 2014, the patient was diagnosed with a large, posterior cranial fossa tumor (Figures 3a and 3b) and underwent a craniotomy; pathology revealed metastatic, poorly differentiated squamous cell carcinoma. The imaging findings were negative for an abdominopelvic recurrence. The patient remained in clinical remission for eight years following her initial diagnosis but ultimately, she died from the neurologic disease in October 2014.

\section{Discussions}

Since locally advanced cervical cancer is potentially difficult to manage, clinicians have endeavored to maximize disease control and improve patient survival. Unfortunately, the relapse rates, especially for FIGO stage IVA cervical cancer, following chemoradiation are high, and patient 5-year overall survival rates are discouraging [2].

Recently, clinical management for advanced cervical cancer has also included targeted therapy, intensitymodulated radiotherapy, dose-dense chemotherapy and minimally invasive surgery [15-17]. However, despite the advances in chemotherapy and radiotherapy, random- 


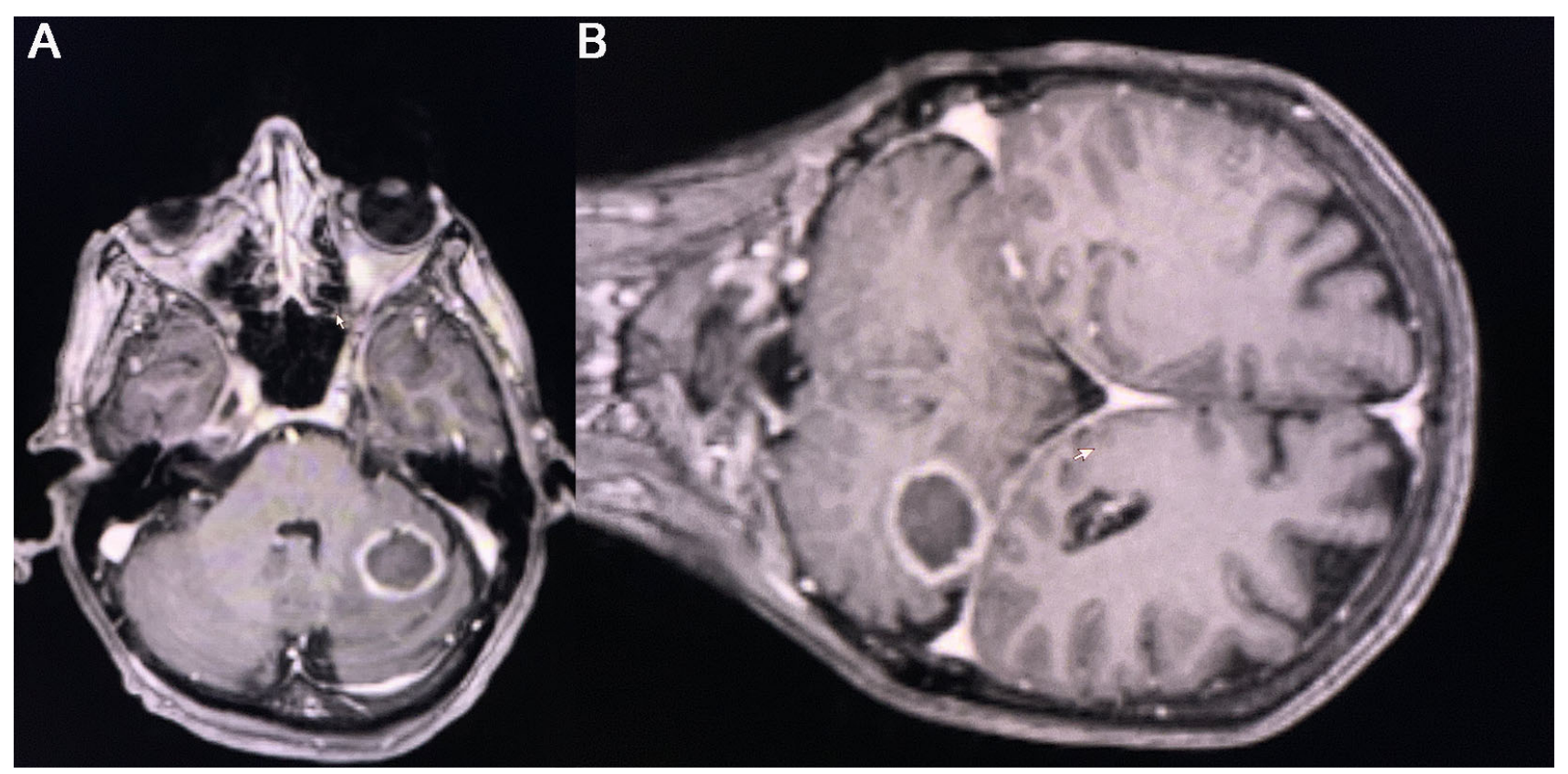

Figure 3. - August 2014 MRI axial (Figure 3A) and coronal (Figure 3B) T1-weighted scan displaying the posterior cranial fossa lesion.

ized trials have not demonstrated their superiority over surgery and chemotherapy [18]; thus, if indicated, the role of surgery for stage IVA cervical carcinoma should be considered a viable option in patients for whom the tumor is amenable to surgical resection $[13,14]$.

In the present case study, we recount the long-term history of a stage IVA cervical cancer patient who presented with a large, $7 \mathrm{~cm}$ cervical tumor that included bladder invasion. Following neoadjuvant chemotherapy, an extended radical hysterectomy and chemoradiation, the patient's disease remained in clinical remission for over 5 years. Since the initial results described in locally advanced cervical cancer trials [19], the utility of a radical hysterectomy has been impugned, primarily because of unimproved patient survival rates and the elevated incidence of post-operative bowel and bladder morbidity [20]. Instead, a radical hysterectomy is often reserved for those patients who have residual disease or fail chemoradiation, although this procedure does not appear to improve survival outcomes [14, 21]; one study indicated that there was only a $3 \%$ survival benefit from a radical hysterectomy for patients with relapsed III-IVA disease [14].

In this case study, the stage IVA cervical cancer patient was managed with carboplatin, paclitaxel and bevacizumab to address her disease recurrence although we recognize that paclitaxel and cisplatin are the standard of care [22] although we recognize that paclitaxel and cisplatin are the standard of care [23]. We also treated the patient with paclitaxel maintenance therapy to further prolong the response [24]. Nevertheless, there is cumulative toxicity inherent in chemoradiation, namely bowel necrosis, myelosuppression and neurotoxicity [25], the latter of which was potentially mitigated by the reduced dose of paclitaxel [26].
Prior studies employing neoadjuvant chemotherapy and radical hysterectomy have documented 5-year OS rates of $90.8 \%, 72.6 \%$, and $52.5 \%$ for FIGO stage IB2-IIA, IIB, and III, respectively [27]; since current 5-year survival rates for stage IVA cervical carcinoma are nearly $16 \%$ [2], perhaps neoadjuvant chemotherapy and radical hysterectomy should be further evaluated in the management of this disease.

When considering stage IVA cervical cancer, surgically addressing rectal involvement is theoretically easier than bladder invasion. Specifically, posterior stage IVA patients can be treated with a primary posterior exenteration and low rectal reanastomosis, on which we previously reported with a stage IIIB cervical carcinoma patient who remained in clinical remission for eight years [28]. However, a pelvic exenteration is associated with high morbidity and mortality rates [29].

We acknowledge that the inclusion of a radical hysterectomy in first line treatment of locally advanced cervical carcinoma is not considered standard of care. We also recognize that the patient's beneficial outcome may have been attributed to chemoradiation alone (i.e., the surgery's impact on survival may have been inconsequential) or a favorable tumor biology. Nevertheless, surgery after neoadjuvant therapy for advanced cervical cancer potentially results in acceptable morbidity and facilitates a pathological response, which may also improve disease free survival [6, 7].

Despite the NCCN guidelines for managing locally advanced cervical carcinoma, one may conjecture that initially resecting aggressive cervical carcinoma lesions wherein the prognosis is very unfavorable (e.g., stage IVA disease) potentially confers improved patient outcomes. 
Additional study incorporating the impact of an extended radical hysterectomy into standard therapy for highly selected stage IIB, IIIB and IVA cancers on survival is warranted.

\section{Author contributions}

JM and MAR contributed significantly to the study's initiation, development and manuscript revision. BG substantially contributed to the study's development and manuscript revision. HE conducted the review of the patient's pathologic diagnosis and treatment history and also substantially contributed to the study's development and manuscript revision. MCR was instrumental in reviewing the patient's charts, conducting the radiologic evaluation and revising the manuscript. RB reviewed the patient's chart, analyzed the chemotherapy treatment lines and corresponding outcomes, and significantly assisted with the manuscript development and revision.

\section{Funding sources}

This case study was supported by the Women's Cancer Research Foundation and Joan \& Len Rullo in memory of Elizabeth Johnson.

\section{Ethics approval and consent to participate}

The authors received verbal consent to publish this case report. Additionally, the case report was prepared in accordance with HIPAA requirements and privacy regulations.

\section{Conflict of Interest}

All authors deny any conflict of interest associated with this manuscript.

Submitted: October 23, 2019

Accepted: June 04, 2020

Published: October 15, 2020

\section{References}

[1] Siegel R.L., Miller K.D., Jemal A.: "Cancer statistics, 2018". CA Cancer J. Clin., 2018; 68, 7-30.

[2] Razzaghi H., Epidemic Intelligence Service Program, Centers for Disease Control and Prevention; Atlanta Georgia, Saraiya M., Thompson T.D., Henley S.J., Viens L., et al.: "Five-year relative survival for human papillomavirus-associated cancer sites". Cancer, 2018, 124, 203-211.

[3] Monk B.J., Sill M.W., McMeekin D.S., Cohn D.E., Ramondetta L.M., Boardman C.H., et al.: "Phase III Trial of Four CisplatinContaining Doublet Combinations in Stage IVB, Recurrent, or Persistent Cervical Carcinoma: A Gynecologic Oncology Group Study”. J. Clin. Oncol., 2009, 27, 4649-4655.

[4] Rosen V.M., Guerra I., McCormack M., Nogueira-Rodrigues A., Sasse A., Munk V.C. et al.: "Systematic Review and Network MetaAnalysis of Bevacizumab Plus First-Line Topotecan-Paclitaxel or Cisplatin-Paclitaxel Versus Non-Bevacizumab-Containing Therapies in Persistent, Recurrent, or Metastatic Cervical Cancer". Int. J. Gynecol., 2018, 27, 1237-1246.

[5] Sebastião A.M., da Silva Rocha L.S., Gimenez R.D., de Barros L.A.B., Fukushima J.T., da Silva S.C.S., et al.: "Carboplatin-based chemoradiotherapy in advanced cervical cancer: an alternative to cisplatin-based regimen?". Eur. J. Obstet. Gynecol. Reprod. Biol., 2017, 201, 161-165.
[6] Lèguevaque P., Motton S., Delannes M., Querleu D., Soulé-Tholy M., Tap G., et al.: "Completion surgery or not after concurrent chemoradiotherapy for locally advanced cervical cancer?". Eur. J. Obstet. Gynecol. Rep. Biol., 2011, 155, 188-192.

[7] Classe J.M., Rauch P., Rodier J.F., Morice P., Stoeckle E., Lasry S., et al.: "Surgery after concurrent chemoradiotherapy and brachytherapy for the treatment of advanced cervical cancer: Morbidity and outcome: Results of a multicenter study of the GCCLCC (Groupe des Chirurgiens de Centre de Lutte Contre le Cancer)". Gynecol. Oncol., 2006, 102, 523-529.

[8] Ferrandina G., Margariti P.A., Smaniotto D., Petrillo M., Salerno M.G., Fagotti A., et al.: "Long-term analysis of clinical outcome and complications in locally advanced cervical cancer patients administered concomitant chemoradiation followed by radical surgery". Gynecol. Oncol., 2010, 119, 404-410.

[9] Touboul C., Uzan C., Mauguen A., Gouy S., Rey A., Pautier P., et al.: "Prognostic Factors and Morbidities After Completion Surgery in Patients Undergoing Initial Chemoradiation Therapy for Locally Advanced Cervical Cancer". the Oncologist, 2010, 15, 405-415.

[10] Wakatsuki M., Kato S., Kiyohara H., Ohno T., Karasawa K., Tamaki T., et al.: "The prognostic value of rectal invasion for stage IVA uterine cervical cancer treated with radiation therapy". Bmc. Cancer, 2016, 16, 244.

[11] Platt S.L., Patel A., Humphrey P.J., Al-Booz H.and Bailey J.: "Completion surgery after chemoradiotherapy for cervical cancer - is there a role? UK Cancer Centre experience of hysterectomy post chemo-radiotherapy treatment for cervical cancer". J. Obstet. Gynaecol., 2019, 39, 68-73.

[12] Vale C., Tierney J.F., Stewart L.A., Brady M., Dinshaw K., Jakobsen A., et al.: "Reducing uncertainties about the effects of chemoradiotherapy for cervical cancer: a systematic review and meta-analysis of individual patient data from 18 randomized trials". J. Clin. Oncol., 2009, 26, 5802-5812.

[13] Marin F., Plesca M., Bordea C.I., Moga M.A., Blidaru A.: “Types of radical hysterectomies : From Thoma Ionescu and Wertheim to present day". J. Med. Life, 2015, 7, 172-176.

[14] Benedetti Panici P., Bellati F., Manci N., Pernice M., Plotti F., Di Donato V., et al.: "Neoadjuvant Chemotherapy Followed by Radical Surgery in Patients Affected by FIGO Stage IVA Cervical Cancer". Ann. Surg. Oncol., 2007, 14, 2643-2648.

[15] Gallotta V., Chiantera V., Conte C., Vizzielli G., Fagotti A., Nero C., et al:: "Robotic Radical Hysterectomy After Concomitant Chemoradiation in Locally Advanced Cervical Cancer: A Prospective Phase II Study". J. Minim. Invasive Gynecol., 2017, 24, 133-139.

[16] Ferrandina G., Gambacorta A., Gallotta V., Smaniotto D., Fagotti A., Tagliaferri L., et al.: "Chemoradiation With Concomitant Boosts Followed by Radical Surgery in Locally Advanced Cervical Cancer: Long-term Results of the ROMA-2 Prospective Phase 2 Study". Int. J. Radiat. Oncol. Biol. Phys., 2014, 90, 778-785.

[17] Ferrandina G., Palluzzi E., Gallotta V., Gambacorta M.A., Autorino R., Turco L.C., et al.: "Neo-adjuvant platinum-based chemotherapy followed by chemoradiation and radical surgery in locally advanced cervical cancer (Lacc) patients: A phase II study". Eur. J. Surg. Oncol., 2018, 44, 1062-1068.

[18] Fanfani F., Vizza E., Landoni F., de Iaco P., Ferrandina G., Corrado G., et al:: "Radical hysterectomy after chemoradiation in FIGO stage III cervical cancer patients versus chemoradiation and brachytherapy: Complications and 3-years survival". Eur. J. Surg. Oncol., 2016, 42, 1519-1525.

[19] Keys H.M., Bundy B.N., Stehman F.B., Muderspach L.I., Chafe W.E., Suggs C.L., 3rd. et al.: "Cisplatin, radiation and adjuvant hysterectomy compared with radiation and adjuvant hysterectomy for bulky stage". IB cervical carcinoma. NEJM., 1999, 340, 1154-1161.

[20] Zigras T., Lennox G., Willows K. Covens A.: "Early Cervical Cancer: Current Dilemmas of Staging and Surgery". Curr. Oncol. Rep., $2017,19,51$.

[21] Marchetti C., De Felice F., Di Pinto A., Romito A., Musella A., Palaia I., et al. Marchetti C., De Felice F., Di Pinto A., Romito A., Musella A., Palaia I., et al.: "Survival Nomograms after Curative Neoadjuvant Chemotherapy and Radical Surgery for Stage IB2-IIIB Cervical Cancer". Cancer Res. Treat., 2018, 50, 768-776.

[22] Lorusso D., Petrelli F., Coinu A., Raspagliesi F. Barni S.: "A systematic review comparing cisplatin and carboplatin plus paclitaxelbased chemotherapy for recurrent or metastatic cervical cancer". Gynecol. Oncol., 2014, 133, 117-123. 
[23] Kitagawa R., Katsumata N., Shibata T., Kamura T., Kasamatsu T., Nakanishi T., Nishimura S., et al.: "Paclitaxel Plus Carboplatin Versus Paclitaxel Plus Cisplatin in Metastatic or Recurrent Cervical Cancer: The Open-Label Randomized Phase III Trial JCOG0505". J. Clin. Oncol., 2015, 33, 2129-2135.

[24] Micha J. P., Sassoon A.F., Wong H. and Goldstein B.H.: "Prolonged remission of recurrent cervical carcinoma following paclitaxel and carboplatin chemotherapy with paclitaxel maintenance chemotherapy". Anticancer. Drugs, 2015, 26, 793-796.

[25] Giavedoni M.E., Staringer L., Garrido R., Bertoncini C., Sardi M., Perrotta M. et al.: "Experience with concurrent chemoradiotherapy treatment in advanced cervical cancer: results from a hospital in Argentina". Ecancermedicalscience, 2019, 13, 919.

[26] Abaid L.N., Micha J.P., Rettenmaier M.A., Brown J.V., Mendivil A.A., Lopez K.L., et al.: "A phase II study of modified dosedense paclitaxel and every 4-week carboplatin for the treatment of advanced-stage primary epithelial ovarian, fallopian tube, or peritoneal carcinoma". Cancer Chemother. Pharmacol., 2013, 72, 101107.

[27] Rose P.G., Java J., Whitney C.W., Stehman F.B., Lanciano R., Thomas G.M., et al.: "Nomograms Predicting Progression-Free
Survival, Overall Survival, and Pelvic Recurrence in Locally Advanced Cervical Cancer Developed From an Analysis of Identifiable Prognostic Factors in Patients From NRG OncologyGynecologic Oncology Group Randomized Trials of Chemoradiotherapy". J. Clin. Oncol., 2015, 33, 2136-2142.

[28] Micha J.P., Brown J.V.: "Primary Surgical Cure of Stage III-B Cervical Carcinoma with Adnexal Metastasis”. Gynecol. Oncol., 1998, 71, 317-319.

[29] Matsuo K., Mandelbaum R.S., Adams C.L., Roman L.D.and Wright J.D.: "Performance and outcome of pelvic exenteration for gynecologic malignancies: A population-based study". Gynecol. Oncol., $2019,153,368-375$.

\section{Corresponding Author:}

BRAM GOLDSTEIN, Ph.D.

Women's Cancer Research Foundation, 351

Hospital Road,

Suite \#506, Newport Beach, CA 92663 (USA)

Email: bram@gynoncology.com 